\title{
24 hours prehabilitation for thoracoscopic surgery; is it enough to improve outcome?
}

\author{
Nafady $\mathrm{M}^{1}$, Elkhayat $\mathrm{H}^{2 *}$, Azer $\mathrm{SZ}^{1}$ and Mohammed $\mathrm{MA}^{1}$ \\ ${ }^{1}$ Medical-Surgical Nursing, Faculty of Nursing, Assiut University, Egypt \\ ${ }^{2}$ Cardiothoracic-Surgery, Faculty of Medicine, Assiut University, Egypt
}

\begin{abstract}
Background: VATS is used in both diagnostic and therapeutic pleural, lung and mediastinal surgery.

Objective: assess the patients' knowledge about thoracoscopic surgery and evaluate the effect of 24 hours prehabilitation protocol on patients' length of hospital stay and quality of life.

Patient and method: Quasi experimental research design was used to conduct this study. The sample was consisted of a convenience sixty adult patients undergone thoracoscopic surgery. The following tools were utilized for data collection: “An interview questionnaire sheet for patients" and” World Health Organization Quality
\end{abstract} of Life".

Result: There was significant difference between study and control groups as regard to hospital stay with no significant difference as regard total quality of life.

Conclusion: 24 hours prehabilitation for thoracoscopic surgery should be tried in centers with no long wait list and can lead to shorter hospital stay.

\section{Introduction}

During the last three decades, video-assisted thoracoscopic surgery (VATS) has revolutionized the surgical approach to several thoracic pathologies. Compared to thoracotomy, VATS has demonstrated not only a significant reduction in pain, recovery time and complications but also an improvement in the post-operative quality of life for patients. Therefore, VATS should be considered the conventional approach for almost all common thoracic operations [1].

VATS is principally employed in the management of pulmonary, mediastinal, and pleural pathology. Its main benefit has been the avoidance of a thoracotomy incision, which allows a shorter operating time, less postoperative morbidity, and earlier return to normal activity than can be achieved with thoracotomy [2].

Enhanced recovery after surgery (ERAS) programs has been focused mainly in minimal invasive surgical approach during lung resection and respiratory rehabilitation after surgery. Prehabilitation has demonstrated reduction of morbidity-mortality in other surgeries but in thoracic surgery continues to be under discussion. The implementation of a preoperative respiratory rehabilitation could optimize patient's physical capacity before surgery, improve outcomes and enhance recovery [3]

High-intensity interval training has been assumed as the best alternative option to endurance training, with duration of the intervention from 2 to 6 weeks. It continues a limitation in some countries where patients can be operated in less than 2 weeks, but generally 4 weeks of waiting is the usual and scientific societies support these periods of time [3].

Prehabilitation has an impact on the occurrence and severity of postoperative complications. Prehabilitation is easy to perform and easily adapted to each patient's functional abilities. Early recovery of physical function is possible after VATS but does not necessarily correlate with early QOL recovery. It is therefore necessary to perform perioperative interventions to quickly restore QOL after surgery [4].

Nowadays, healthcare system is facing numerous challenges due to aging population, medical technique advancement and rise of consumerism. Surgical technique and treatment strategies for thoracic surgical patients continue to evolve. Nurses, as one of the pivotal members of the healthcare teams, need to grow and go forward together as well. Thoracic nursing team works closely together with other healthcare professionals and provides quality care to patients suffering from thoracic diseases. Post-operative nursing interventions including complication monitoring and early mobilization remain the most important and essential care to ensure early and complicationfree rehabilitation [5].

High-risk patients can be optimized with preoperative and postoperative cardiopulmonary rehabilitation to reduce their operative risk, frequency of complications and hospital stay and improve postoperative outcomes including postoperative lung function. In addition, preoperative pulmonary rehabilitation may improve preoperative exercise capacity and so operability. The future development and adoption of innovative strategies is required to reduce

*Correspondence to: Elkhayat $\mathrm{H}$, Cardiothoracic surgery department, Assiut university hospitals, Assiut, Egypt, Tel: +201005549653; Fax: +20882333327; E-mail: dr_khayat@hotmail.com

Key words: prehabilitation protocol, quality of life, thoracoscopic surgery

Received: May 14, 2019; Accepted: May 24, 2019; Published: May 27, 2019 
the impact of post-operative complications in an ageing co morbid population [6].

\section{Material and method}

This was a prospective randomized case-control pilot clinical trial (clinical trial number: NCT03915093) carried out from January 2018 till December 2018 on patients who met the inclusion criteria. Quasi experimental research design was utilized to conduct this study.

The sample size was 73 patients per year was selected by using the equation according to Steven Thompson [7].

\section{Inclusion criteria:}

- Age between 18-65 year.

- All cases undergone video thoracoscopic surgery.

- Patients accept to continue with the researcher until follow up.

\section{Exclusion criteria:}

- Converted to thoracotomy.

- Patients refuse to participate.

\section{World Health Organization Quality of Life (WHOQOL)}

WHOQOL [8] contains 5 broad domains of QOL within which 18 facets are covered to determine the quality of life. These 5 domains include physical, social, psychological, environmental and perceived QOL.

\section{Distribution of the scores in QOL tool}

The scoring of these variables, a 3-point lickert scale on tables was adopted for the answer low $\mathrm{QOL}=0-3$, moderate $\mathrm{QOL}=4-6$, and high $\mathrm{QOL}=7-9$.

Data were collected at the cardiothoracic surgery department at Assiut University Hospital. The tools were filled through interviewing and the purpose of the study was explained to all patients prior to collection of data.

For the control group; after taking the patient oral agreement for voluntary participation in the study, the researcher then fills the patient's structured assessment sheet. After the patient's discharge from the hospital, the researcher calls the patient for follow up in telephone one-month post thoracoscopic surgery for re-evaluating the patient's condition includes quality of life.

For the study group; after filling the patient's structured assessment sheet, the researcher explains to the patient prehabilitation protocol in the following sequence: the prehabilitation protocol was prepared in a simple Arabic language with a simple photo illustrations and contain information about thoracoscopic surgery (pre - post care and follow up instructions). It was administered to the patient in two sessions, the duration of each session was about 45 minutes, including 15 minutes for discussion and feedback, but the time may differ from one patient to another according to educational level and his $\backslash$ her ability to understand what is being said. Each patient in the study group obtained a copy of the prehabilitation booklet. After the patient's discharge from the hospital, the researcher calls the patient for follow up in telephone one-month post thoracoscopic surgery for re-evaluating the patient's condition include (knowledge and quality of life).

\section{Content validity}

The content validity for the booklet in Arabic language was done by 5 expertise (medical staff) from cardiothoracic surgery department \& (nursing staff) from the medical-surgical nursing department who reviewed the booklet for clarity, relevance, comprehensiveness, understanding, applicability and easiness.

\section{Results}

During the study time frame, the study included 73 patients, 13 of whom were excluded from the study, 3 of them converted to thoracotomy and the other 10 not respond to telephone calls. Finally, 60 patients were completely followed up and analyzed. Two groups were formed: one group with prehabilitation $(n=30)$ and one group without prehabilitation $(n=30)$. Patients were followed from $24^{\text {th }}$ hour preoperatively to the postoperative day 30 .

More than half of the study group patients (53.3\%) and more than one third of the control patients (33.3\%) their age ranged from (1829 years). About two thirds of both groups (study $63.3 \%$ and control $66.7 \%$ ) were male. As regarding to level of education the highest percentage in study group (36.7\%) were highly educated and in control group (33.3\%) were not educated.

There was no significant difference between both groups as regarding to type of operation with the highest percentage underwent lung resection surgery in both groups (36.7\% and $33.3 \%)$ respectively (Table 1).

There was statistical significance difference between study and control group patients as regarding to environmental and general or perceived QOL domains and there was no significance difference between study and control group patients as regarding to physical, psychological and social domains (Table 2).

This study presented that, there was significant difference between study and control groups as regard to hospital stay with means (3.166 \pm 2.018 and $5.033 \pm 4.649$ ) respectively.

Table 1. Frequency distribution of both groups (study and control) in relation to types of operation $(\mathrm{n}=60)$

\begin{tabular}{|c|c|c|c|c|c|}
\hline \multirow{2}{*}{ Variables } & \multicolumn{2}{|c|}{ Study } & \multicolumn{2}{|c|}{ Control } & \multirow{2}{*}{ P.V } \\
\hline & $\mathbf{N}$ & $\%$ & $\mathbf{N}$ & $\%$ & \\
\hline Sympathectomy & 5 & 16.7 & 2 & 6.7 & \multirow{5}{*}{$.714 \mathrm{~ns}$} \\
\hline Lung resection & 11 & 36.7 & 10 & 33.3 & \\
\hline Pleural or lymph node biopsy & 6 & 20.0 & 8 & 26.7 & \\
\hline Decortication & 5 & 16.7 & 5 & 16.7 & \\
\hline Others & 3 & 10.0 & 5 & 16.7 & \\
\hline
\end{tabular}

Chi-Square Tests

Ns $=$ Non-significant difference $\mathrm{p}<0.05$

Table 2. Mean total quality of life for studied patients (study and control groups) as regarding to general domains $(n=60)$

\begin{tabular}{|c|c|c|c|c|}
\hline \multirow{2}{*}{ Variables } & Total score & Study & Control & \multirow{2}{*}{ P.V } \\
\cline { 3 - 5 } & Means \pm SD & Means \pm SD & \\
\hline Physical & 63 & $37.800 \pm 2.524$ & $38.000 \pm 2.228$ & $.746 \mathrm{~ns}$ \\
\hline Psychological & 36 & $29.600 \pm 1.003$ & $28.900 \pm 2.073$ & $.101 \mathrm{~ns}$ \\
\hline Social & 27 & $11.833 \pm 11.286$ & $14.166 \pm 10.989$ & $.421 \mathrm{~ns}$ \\
\hline Environmental & 27 & $20.733 \pm 2.196$ & $19.633 \pm 1.973$ & $.046^{*}$ \\
\hline Perceived QOL & 9 & $7.733 \pm 1.48$ & $6.933 \pm 1.172$ & $.024^{*}$ \\
\hline $\begin{array}{c}\text { Total score } \\
\text { QOL }\end{array}$ & 162 & $107.700 \pm 11.614$ & $107.633 \pm 10.707$ & $.982 \mathrm{~ns}$ \\
\hline
\end{tabular}

Independent t-test, $*=$ Significant difference, $* \mathrm{p} \leq 0.05, \mathrm{Ns}=$ Non-significant difference $\mathrm{p}<0.05, * *=$ highly significance, $\mathrm{p} \leq 0.01$ 


\section{Discussion}

Based on the results of the present study; more than half of the study group patients and more than third of the control group patients their age ranged from (18-29 years). Most of both groups were male. As regarding to level of education the highest percentage in study group were highly educated and in control group were not educated.

Schwarzbach et al. [9] unlike our study as regard age of patients; they stated that the median age was 66 year in study group and 60 year in control group, but they agree with the study as regard to sex which revealed that the majority of patients were male. They reported that there are no statistically significant differences in sex, age between both groups. This study was in the same line with Hammad and Saad [10] who found that, the mean age of the patients was 27.8 years (range 1935).

As regarding to diagnosis, it was noticed that the highest percentage of patients had lung, mediastinal or pleural mass with no significant difference between both groups as regarding to type of operation with the highest percentage underwent lung resection surgery in both groups.

These results in the same line with Schwarzbach et al. [9] who reported that the majority of patients in both groups underwent VATS for diagnostic or therapeutically pulmonary wedge resection (pulmonary/pleural mass or bullae resection). The most common underlying diagnoses were malignancies in both groups.

The present study revealed that, there was highly statistical significance difference between study group patients pretest and posttest in total knowledge. This result was supported by Brunetti et al. [11] who told that preoperative educational program helps to set expectations about surgical and anesthetic procedures and may diminish fear, fatigue and pain and enhance recovery and early discharge. Verbalized education, leaflets and multimedia information containing explanations of the procedure and cognitive interventions may improve pain control, nausea and anxiety after surgery.

Refai et al. [12] revealed that, a preoperative personal education may play a key role to reduce stress, fear or anxiety and improve the morbidity of patients, enabling them to achieve functional and psychological compensatory mechanisms more quickly. Preoperative patient counseling performed using verbal, written or multimedia materials to make the patient a potentially active participant and the main character of recovery, able to positively impact self throughout the surgical and healing process.

Sebio Garcia et al. [13] reported that education provides beneficial effects with no evidence of harm. Pain control appears better following lung resection. It is recommended that patients should routinely receive dedicated preoperative education and concluded that prehabilitation is beneficial, but, because of study heterogeneity, the exact duration, intensity, structure and patient selection to achieve maximum efficacy is uncertain [13].

Boujibar et al. [14] reported that physical activities are method of disease control and treatment for patients. In particular, exercise has positive effects on physical and mental health and is essential for patients who feel their quality of life has deteriorated due to physical, psychological, emotional, and financial hardships [14].

Batchelor et al. [15] mentioned that preoperative exercise rehabilitation program can reduce hospital stay and postoperative pulmonary complications. Because of study heterogeneity, no firm recommendations can be made on the nature of the intervention in terms of exercise modality, delivery and frequency or preoperative duration. Preoperative exercise program should be considered for patients with borderline lung function or exercise capacity.

Refai et al. [12] revealed that, the information transfer may be impaired by the patient's intellectual level, language barriers, learning disabilities, and cultural barriers. Considering all these factors, questions and discussions with the surgeon should be encouraged as an additional tool provided to the patients [12].

According to Schwarzbach et al. [9] reported that length of hospital stay (LOS) was 9 days for clinical pathway (CP) group and 14 days for pre pathway group. The median length of stay in the hospital was five days shorter in the CP group as compared to the pre pathway group [9]. This was confirmed by the result of the present study which illustrated that the means of hospital stay for study and control groups were $(3.166 \pm 2.018$ and $5.033 \pm 4.649)$ respectively.

This study also, supported by Gao et al. [16] who reported a correlation between prehabilitation and length of hospital stay, but dis agree with Boujibar et al. [14] who found that there was a median difference of two days in LOS between groups, but this was not statistically significant [14].

Valenzuela et al. [17] in the same line with the current study who mentioned that shorter hospital stays with lower postoperative complications were found in the preoperative physiotherapy education group, the differences were not significant. There was no significant differences in the types of complications between the groups ( $p>0.05)$.

Bertolaccini et al. [18] found that, depending on the type of surgical procedure, most patients stay in the hospital one to four days and can return to their normal activity levels within four weeks. VATS results in less pain during weeks and months of recovery; patients have much smaller scars; and patients have a better chance of breathing normally post-surgery.

This study clarified that, there was significance difference between study and control groups' patients as regarding to environmental and general or perceived QOL domains and there was no significance difference between study and control group patients as regarding to physical, psychological and social domains with better improvements in total QOL of study group than control group.

Sanchez-Lorente et al. [19] reported that VATS has been shown to deliver a better immune system response, provide a better chance of breathing normally and a better quality of life after surgery.

Anami et al. [20] reported that early recovery of physical function is possible after VATS but does not necessarily correlate with early QOL recovery. It is therefore necessary to perform perioperative interventions to promptly restore QOL after surgery. Shi et al. [21] found that patients who underwent VATS returned to their baseline activity, mood and enjoyment of life at a significantly faster rate than patients who underwent open lobectomy.

White et al. [22] found that, preoperative education programs make patients would no longer arrive at the operating theatre frightened and unaware of what will happen to them. The provision of good quality preoperative information facilitates patient's active involvement in their care and may contribute to an overall increase in satisfaction. Preadmission information intervention helps reduce postoperative pain levels and significantly increases knowledge of self-care and complication management. 
Mielck et al. [23] found that less than half of the studied sample has low evaluation of the quality of life and which are responsible for poor community health and low satisfied about their health with chronic disease.

Deasy et al. [24] revealed that perioperative rehabilitation strategies recommended to prevent postoperative complications include lung expansion interventions, deep breathing exercises, incentive spirometry, and ambulation. Negative automatic thoughts are associated with reduced health behavior and physical activity.

Ronco et al. [25] reported that surgical patient outcomes are improved by patient education, and the maladaptive coping style is a risk factor for psychiatric morbidity and decreased survival in cancer patients.

Öz et al. [26] revealed that thoracic surgery operations caused substantial dissatisfaction in life comfort especially in the third month postoperatively. The worsening in physical function, physical role, pain and mental health is much more in patients with resection compared with the patients who did not undergo resection.

Möller et al. [27] found that the extent of surgery, age, and adjuvant therapy, was predictive of a reduced postoperative QOL 6 months after lung surgery. Other factors, i.e., gender, comorbidity, postoperative complications, and tumor stage, were not associated with a higher risk for impaired postoperative QOL.

\section{Conclusion}

The result of the present study concluded that; 24 hours prehabilitation for thoracoscopic surgery should be tried in centers with no long wait list and can lead to shorter hospital stay and improvements in total knowledge and total QOL after prehabilitation protocol implementation.

\section{References}

1. Petersen RH, Holbek BL, Hansen HJ, Kehlet H (2017) Video-assisted thoracoscopic surgery-taking a step into the future. Eur J Cardiothorac Surg 51: 694-695. [Crossref]

2. Jiang N, Lu Y, Wang J (2019) Is single-port video-assisted thoracic surgery for mediastinal cystectomy feasible? J Cardiothorac Surg 14: 18. [Crossref]

3. Sanchez-Lorente D, Navarro-Ripoll R, Guzman R, Moises J, Gimeno E, et al. (2018) Prehabilitation in thoracic surgery. J Thorac Dis 10: S2593-2593S2600. [Crossref]

4. Yeung WW (2016) Post-operative care to promote recovery for thoracic surgical patients: a nursing perspective. J Thorac Dis 8: S71-S77. [Crossref]

5. Ljungqvist O, Scott M, Fearon KC (2017) Enhanced Recovery After Surgery: A Review. JAMA Surg 152: 292-298. [Crossref]

6. Agostini P, Lugg ST, Adams K, Vartsaba N, Kalkat MS, et al. (2017) Postoperative pulmonary complications and rehabilitation requirements following lobectomy: a propensity score matched study of patients undergoing video-assisted thoracoscopic surgery versus thoracotomy. Interact Cardiovasc Thorac Surg 24: 931-937. [Crossref]

7. Steven K, Thompson (2012) sampling, Wiley series in probability and statistics, NY Wiley: 59-60.

8. Kane R (2003) Definition Measurement and Correlate of Quality of Life in Nursing Homes: Toward a Reasonable Practice, Research and Policy Agenda. Gerontologist 43: 28-36. [Crossref]
9. Schwarzbach MH, Ronellenfitsch U, Wang Q, Rössner ED, Denz C, et al. (2010) Effects of a clinical pathway for video-assisted thoracoscopic surgery (VATS) on quality and cost of care. Langenbecks Arch Surg 395: 333-340. [Crossref]

10. Hammad AM, Saad M (2012) Life quality after video-assisted thoracoscopic thoracic sympathetic chain clipping for patients with isolated primary palmar hyperhidrosis. $J$ Arab Society Med Res 7: 63-67.

11. Brunetti M, Shemilt I, Pregno S, Vale L, Oxman AD, et al. (2013) GRADE guidelines: 10. Considering resource use and rating the quality of economic evidence. $J$ Clin Epidemiol 66: 140-50. [Crossref]

12. Refai M, Andolfi M, Gentili P, Pelusi G, Manzotti F, et al. (2018) Enhanced recovery after thoracic surgery: patient information and care-plans. $J$ Thorac Dis 10: S512. [Crossref]

13. Sebio Garcia R, Yanez Brage MI, Gimenez Moolhuyzen E, Granger CL, Denehy L (2016) Functional and postoperative outcomes after preoperative exercise training in patients with lung cancer: a systematic review and meta-analysis. Interact CardioVasc Thorac Surg 23: 486-97. [Crossref]

14. Boujibar F, Bonnevie T, Debeaumont D, Bubenheim M, Cuvellier A, et al. (2018) Impact of prehabilitation on morbidity and mortality after pulmonary lobectomy by minimally invasive surgery: a cohort study. J Thorac Dis 10: 2240-2248. [Crossref]

15. Batchelor TJ, Rasburn NJ, Abdelnour-Berchtold E, Brunelli A, Cerfolio RJ, Gonzalez M, et al. (2019) Guidelines for enhanced recovery after lung surgery: recommendations of the Enhanced Recovery After Surgery (ERAS $($ ) Society and the European Society of Thoracic Surgeons (ESTS). Eur J Cardiothorac Surg 55: 91-115. [Crossref]

16. Gao K, Yu PM, Su JH, He CQ, Liu LX, et al. (2015) Cardiopulmonary exercise testing screening and pre-operative pulmonary rehabilitation reduce postoperative complications and improve fast-track recovery after lung cancer surgery: A study for 342 cases. Thorac Cancer 6: 443-449. [Crossref]

17. Valenzuela FQ, Fernández IP, Cirre MD, Sánchez-Palencia A, de Guevara AC (2017) Impact of major video-assisted thoracoscopic surgery on care quality. $J$ Thorac Dis 9: 4454-4460. [Crossref]

18. Bertolaccini L, Viti A, Terzi A, Rocco G (2016) Geometric and ergonomic characteristics of the uniportal video-assisted thoracoscopic surgery (VATS) approach. Ann Cardiothorac Surg 5: 118. [Crossref]

19. Sanchez-Lorente D, Guzman R, Boada M, Carriel N, Guirao A, et al. (2018) Is it appropriate to perform video-assisted thoracoscopic surgery for advanced lung cancer? Future Oncol 14: 29-31. [Crossref]

20. Anami K, Horie J, Hirayama Y, Yamashita N, Ito K (2018) Changes in exercise tolerance and quality of life are unrelated in lung cancer survivors who undergo videoassisted thoracic surgery. J Phys Ther Sci 30: 467-473. [Crossref]

21. Shi Q, Wang XS, Vaporciyan AA (2016) Patient-Reported Symptom Interference as Measure of Postsurgery Functional Recovery in Lung Cancer. J Pain Symptom Manage 52: 822-31. [Crossref]

22. White J, Dixon S (2015) Nurse led Patient Education Programme for patients undergoing a lung resection for primary lung cancer. J Thorac Dis 7: S131. [Crossref]

23. Mielck A, Vogelmann M, Leidl R (2014) Health-related quality of life and socioeconomic status: inequalities among adults with a chronic disease. Health Qual Life Outcomes 12: 58. [Crossref]

24. Deasy C, Coughlan B, Pironom J, Jourdan D, Mannix-McNamara P (2014) Psychological distress and coping amongst higher education students: a mixed method enquiry. PLoS One 9: e115193. [Crossref]

25. Ronco M, Iona L, Fabbro C, Bulfone G, Palese A (2012) Patient education outcomes in surgery: a systematic review from 2004 to 2010. Int J Evid Based Health 10: 309323. [Crossref]

26. Öz G, Solak O, Metin M, Esme H, Sayar A (2015) The effects of thoracic surgery operations on quality of life: a multicenter study. Clin Respir J 9: 409-413. [Crossref]

27. Möller A, Sartipy U (2012) Predictors of postoperative quality of life after surgery for lung cancer. J Thorac Oncol 7: 406-411. [Crossref]

Copyright: (2019 Nafady M. This is an open-access article distributed under the terms of the Creative Commons Attribution License, which permits unrestricted use, distribution, and reproduction in any medium, provided the original author and source are credited. 